\title{
Migraine in Patients with Metabolic Syndrome: Is there a Relationship to Leptin?
}

Sherifa A Hamed ${ }^{1 *}$, Manal E Ezz-EI-Deen ${ }^{2}$ and Madleen A Abdou ${ }^{3}$

${ }^{1}$ Department of Neurology, Assiut University Hospital, Assiut, Egypt

${ }^{2}$ Department of Internal Medicine, Assiut University Hospital, Assiut, Egypt

${ }^{3}$ Department of Clinical Pathology, Assiut University Hospital, Assiut, Egypt

\begin{abstract}
Previous studies have reported association of migraine and metabolic syndrome (MS) and between MS and leptin (a protein product of the obesity gene). This study aimed to determine whether there is a link between MS and its components, leptin and migraine and its covariates (frequency and duration), as data regarding this relationship are still sparse or even controversial. This study included 60 patients with MS and comorbid migraine with mean age of $47.83 \pm 7.31$ years. Demographic, anthropometric, clinical and lab characteristics were identified. Serum leptin concentrations were also measured. Nearly $58.33 \%$ had episodic migraine (MoA=44.64\%, MA=16.6\%), 35\% had chronic migraine and $6.67 \%$ had tension type headache (TTH). Obesity, type 2 diabetes mellitus and hypertension and were reported in all patients, of them $80 \%$ had hypertriglyceridemia and/or dyslipidemia, $81.67 \%$ had insulin resistance (IR) and 58.33\% hyperleptinemia. Compared to patients with TTH, patients with migraine had higher measurements for BMI (39.01 \pm 6.05$)$, WC $(P=0.058)$, poor glycemic control $(8.11 \pm 1.22)$, SBP $(P=0.052)$, DBP $(P=0.050)$ and serum levels of LDL-C $(P=0.0001)$, fasting insulin $(P=0.0001)$ and leptin $(P=0.0001)$. Leptin concentrations were found to be positively correlated with BMI $(r=0.547, P=0.008), W C(r=0.445, P=0.002), \mathrm{HbA} 1 \mathrm{c}(r=0.656, P=0.001)$ and fasting insulin $(r=0.613, P=0.008)$. The logistic regression to model leptin and headache parameters (frequency and duration) after adjusting age and sex and leptin levels, were found to correlate with BMI, WC and fasting insulin) but this relationship disappeared after adjustment of these covariates. We conclude that comorbid migraine with MS is related to obesity (total body obesity and abdominal adiposity) and insulin abnormalities after adjustment of other covariates.
\end{abstract}

Keywords: Metabolic syndrome; Obesity; Insulin resistance; Leptin

\section{Abbreviations}

MS: Metabolic Syndrome; T2DM: Type 2 Diabetes Mellitus; HTN: Hypertension; TC: Total cholesterol; TG: Triglycerides; LDL-c: Low Density Lipoprotein Cholesterol; HDL-c: High Density Lipoprotein Cholesterol; SBP: Systolic Blood Pressure; DBP: Diastolic Blood Pressure; TBO: Total Body Obesity; BMI: Body Mass Index; WC: Waist Circumference; IR: Insulin Resistance; FBG: Fasting Blood Glucose; OGTT: Oral Glucose Tolerance Test; HbA1c: Gycolysated Hemoglobin; ICHD: International Classification of Headache Disorders; MA: Migraine with Aura; MoA: Migraine without Aura; ELISA: EnzymeLinked Immunosorbent Assay; HOMA-IR: Homeostasis Model Assessment Equation for Insulin Resistance

\section{Introduction}

Metabolic syndrome (MS) is quite common with an estimated prevalence of $5 \%$ of subjects with normal body weight, $22 \%$ of overweight subjects and $60 \%$ of obese subjects $[1,2]$. MS is defined according to the single global definition promoted by several international societies as a cluster of conditions with the following features [3]: obesity [total body obesity (TBO) which is indicated by elevated body mass index (BMI) and abdominal adiposity which is indicated by increased WC], insulin resistance (IR) which refers to the diminished cell response to insulin in promoting transport of glucose from blood to muscles and other tissues [4], type 2 diabetes mellitus (T2DM), hypertension (HTN), cholesterol abnormalities (hypercholesterolemia and/or dyslipidemia), and an increased risk for clotting. Epidemiological, longitudinal and crosssectional case controlled studies have shown increased prevalence of migraine among patients with MS (10-22.5\%) [5,6]. Migraine is a frequent medical condition with an estimated prevalence of $\sim 12 \%$ of the general population [7]. Migraine is defined according to the definition and criteria of the second edition of the International Classification of Headache Disorders (ICHD-2), as a chronic neurological disorder characterized by episodic attacks of moderate to severe headache and associated symptoms [8]. The highest prevalence occurs between the ages of 25 and 55 years, potentially the most productive period of life [8]. Migraine is divided into six major categories, the two most important of which are: migraine without aura (MoA) and migraine with aura (MA). For MoA, the ICHD-2 requires at least five lifetime headache attacks (often unilateral and pulsating) which accompanied by various combination of features (photophobia/phonophobia and/or nausea/vomiting) which last from 4 to 72 hours. MA is characterized by focal neurological phenomena (aura) that usually proceed, accompany or occur in the absence of headache. Most aura symptoms develop over 5-20 minutes and last 20-60 minutes. In one-third of patients with MA, visual aura is the most common form (hemianopia, a crescent with a bright, ragged edge and scintillations, scotoma, photopsia or phosphenes, fortification spectra and other visual manifestations), followed by sensory symptoms (numbness and tingling or paraesthesia). Hemi-motor weakness, dysphasia and incoordination and other signs of brainstem dysfunctions are less common aura symptoms. Chronic migraine (which evolved from episodic migraine and was termed chronic daily headache in ICHD-1 classification) is defined if the

*Corresponding author: Sherifa Ahmed Hamed, Department of Neurology and Psychiatry, Assiut University Hospital, Assiut, Egypt, P.O.Box 71516 Tel: +2 01115324560; Fax: +2 088 2333327, +2 088 2332278; Email: hamed_sherifa@yahoo.com

Received May 08, 2012; Accepted July 07, 2012; Published July 09, 2012

Citation: Hamed SA, Ezz-El-Deen ME, Abdou MA (2012) Migraine in Patients with Metabolic Syndrome: Is there a Relationship to Leptin? Metabolomics 2:114. doi:10.4172/2153-0769.1000114

Copyright: (c) 2012 Hamed SA, et al. This is an open-access article distributed under the terms of the Creative Commons Attribution License, which permits unrestricted use, distribution, and reproduction in any medium, provided the original author and source are credited. 
migraine headache attacks occurs at frequency of $>15$ days/month in a patient not overusing acute medications [9].

MS and migraine are population prevalent and disabling disorders influenced by multifactorial (complex) genetic, environmental and many modifying risk factors (as sedentary lifestyle and progressive weight gain). However, the mechanisms underlying the pathogenesis of MS-related migraine remain unknown. Several studies reported an association between migraine and MS and its different components as obesity (TBO and abdominal adiposity) [10-13], poor glycemic control [14], IR [15], HTN [16] and hypercholesterolemia/dyslipidemia [17].

Recently, a large number of adipocyte-derived adipokines as adiponectin, leptin, resistin, and retinol binding protein 4 , and the resident macrophage-derived adipokines and cytokines as tumor necrosis factor (TNF- $\alpha$ ) and interleukin 6 (IL-6), have been discovered to play critical roles in variety of endocrine functions which are related to organs such as the brain, liver, pancreas and skeletal muscle to control diverse processes as food intake, energy expenditure, carbohydrate and lipid metabolism, blood pressure, blood coagulation, and inflammation [18]. The dys-regulation of adipokines secretion and action have been found to play an important role in initiation of obesity and its related inflammation, MS, T2DM, inflammatory disorders and vascular disorders [18]

Leptin is the best known adipocyte-derived adipokines and a protein product of the obesity gene [19]. Leptin has been found to be positively and predictively associated with obesity [20-21]. IL-6 (which has an important role in the initiation of obesity-related inflammation) [22], hyperglycemia [23], IR [24,25], HTN [26,27] and lipids [28] which are components of metabolic syndrome. Thus, leptin may form a link between MS and migraine.

\section{Aim of work}

This study aimed to determine whether there is a link between MS and its components, leptin and migraine and its covariates (frequency and duration).

\section{Materials and methods}

\section{Subjects}

This cross-sectional study included consecutive 60 patients with MS and complained of headache. Patients were recruited from the outpatient diabetes clinic of the Internal Medicine department of Assiut University Hospital, Assiut, Egypt throughout a period 6 months (20102011), and evaluated by trained physicians according to a standard protocol for the presence of MS and its components [3]. Forty age, sex, socioeconomic status and educationally matched subjects recruited from the general population were included as healthy controls subjects for comparison. The study protocol was carried out according to the Declaration of Helsinki and was in conformity with the local ethical guidelines and informed written consent was obtained from each participant.

Patients were studied during the headache attack-free periods. Headache was classified according to the criteria of the $2^{\text {nd }}$ edition of the International Headache Society [8]. Accordingly, patients with MS were divided into those with episodic migraine (MA and MoA), chronic migraine (evolved from episodic migraine) and other types of primary headaches (e.g. tension type headache or TTH). According to the frequency of migraine attacks, patients with migraine were divided into two groups: a) high frequency group: $\geq 4$ attacks / month, and b) low-frequency group: $<4$ attacks / month [29].

Excluded were: 1) smokers and alcoholics, 2) patients with other medical, neurologic, psychiatric or systemic diseases as major cardiovascular events (e.g. myocardial infarction, stroke, coronary artery bypass graft/percutaneous transluminal coronary angioplasty revascularization intervention); transient ischemic attacks, cerebrovascular stroke; epilepsy; active gastrointestinal disease; gout; recent infection; chronic inflammation; serum creatinine $>150 \mu \mathrm{mol} / \mathrm{l}$ and malignancies, 3) patients treated with insulin because exogenous insulin might lead to a false high insulin concentration that was used in the calculation of the IR index, 4) patients on regular medications other than non-steroidal anti-inflammatory (NSAIDS), anti-migrainous, anti-diabetic, anti-hypertensive and lipid lowering drugs, and 5) pregnancy, lactation or use of contraceptive pills.

\section{Methods}

All participants were subjected to medical, endocrinological and neurological history and examination. The following demographic, anthropometric, cardiovascular, and clinical parameters were collected per subject: age, gender, weight, height, BMI and WC, duration of illness (years), current anti-diabetic, anti-hypertensive and lipid lowering therapies. The body mass index (BMI) is a commonly used anthropometric measure to estimate total body fat and is often calculated based on patient's self-reported height and weight. According to BMI, subjects are divided into 5 categories: i) underweight $\left(<18.5 \mathrm{~kg} / \mathrm{m}^{2}\right)$, ii) normal weight ( 18.5 to $\left.24.9 \mathrm{~kg} / \mathrm{m}^{2}\right)$, iii) overweight ( 25 to $29.9 \mathrm{~kg} / \mathrm{m}^{2}$ ), iv) obese (30 to $\left.34.9 \mathrm{~kg} / \mathrm{m}^{2}\right)$ and v) morbidly obese $\left(>35 \mathrm{~kg} / \mathrm{m}^{2}\right)$ [30]. Abdominal adiposity was defined when $\mathrm{WC} \geq 94 \mathrm{~cm}$ in males and $\geq 80$ $\mathrm{cm}$ in females [3]. IR is usually calculated using the homeostasis model assessment equation (HOMA-IR) (formula: Fasting insulin ( $\mathrm{uIU} / \mathrm{mL}$ ) $\mathrm{X}$ fasting glucose $(\mathrm{mmol} / \mathrm{L}) / 22.5$. Patients were consider to have IR if HOMA-IR $\geq 2.6$ [31].

\section{Specimen collection and analysis}

No medications were taken on the morning of the study day. Venous blood samples $(6 \mathrm{ml})$ were obtained via venipuncture at 8:00-10:00 a.m. after an overnight fast for at least 12 hours. $2 \mathrm{ml}$ of K3EDTA blood was drawn in a vacutainer tube for immediate assessment of complete blood count (CBC) (Cell Dyn 3500, Abbot Diagnostics, Abbott Laboratories. Abbott Park, Illinois, USA) and preparation of hemolysate was kept frozen for estimation of HbAlc, a clinical indicator of glycemic control. HbAlc determination is based on turbidimetric inhibition immunoassay for hemolyzed whole blood and was measured using Hitachi 911 autoanalyzer (Roche Diagnostics, Mannheim, Germany. The remaining $4 \mathrm{ml}$ were immediately centrifuged at 2,500 rpm for 15 minutes and the obtained serum was divided into 3 aliquots: one was utilized for FBG, kidney, liver functions and lipogram, while the other two aliquots were stored at $-70^{\circ} \mathrm{C}$ for latter assessment of serum insulin and leptin. Serum levels of TC, TG, HDL-c, LDL-c were measured by enzymatic colourimetric method [Hitachi 911 autoanalyzer (Roche Diagnostics, Mannheim, Germany]. Insulin was determined by enzyme-linked immunosorbent assay (ELISA) (Diagnostic Systems Laboratory, Webster, TX, USA). Serum leptin was measured by an ELISA (BioVendor Laboratory Medicine, Inc., Modrice, Czech Republic). Two hours after blood withdrawal for estimation of FBG the patients received formal standard oral glucose and $3 \mathrm{ml}$ blood samples were withdrawn from each participant for estimation of postprandial blood glucose level (i.e. oral glucose tolerance test or OGTT). The measurements of serum insulin and leptin levels were combined 
Citation: Hamed SA, Ezz-El-Deen ME, Abdou MA (2012) Migraine in Patients with Metabolic Syndrome: Is there a Relationship to Leptin? Metabolomics 2:114. doi:10.4172/2153-0769.1000114

Page 3 of 6

\begin{tabular}{|c|c|c|c|c|c|c|}
\hline \multirow[t]{2}{*}{ Demographic and Clinical characteristics } & \multicolumn{2}{|c|}{ Patients $(n=60)$} & \multirow{2}{*}{$\begin{array}{l}\text { Control subjects } \\
\qquad(n=40)\end{array}$} & \multirow[t]{2}{*}{ P1-value } & \multirow[t]{2}{*}{ P2-value } & \multirow[t]{2}{*}{ P3-value } \\
\hline & Patients with migraine & Patients with TTH & & & & \\
\hline Male/female; \# (\%) & $22 / 38$ & $1 / 3$ & $12 / 28$ & - & - & - \\
\hline Age; years & $32.0-55.0(47.83 \pm 7.31)$ & $32.0-55.0(48.55 \pm 7.60)$ & $\begin{array}{c}35.55-55.00(48.35 \pm \\
7.60)\end{array}$ & 0.494 & 0.345 & 0.255 \\
\hline $\begin{array}{l}\text { Patients with episodic migraine; \# (\%) } \\
\text { MoA } \\
\text { MA } \\
\text { Patients with chronic migraine; \# (\%) } \\
\text { Patients with TTH; \# (\%) } \\
\text { Duration of migraine; years } \\
\text { Duration of episodic migraine; years } \\
\text { Duration of chronic migraine; years } \\
\text { Duration of TTH; years }\end{array}$ & $\begin{array}{c}35(62.5 \%) \\
25(44.64 \%) \\
10(17.86 \%) \\
21(37.5 \%) \\
4(6.67 \%) \\
1.00-22.00(8.528 \pm 4.27) \\
2.0-18.0(6.56 \pm 1.45) \\
1.00-22.00(9.328 \pm 4.27) \\
-\end{array}$ & $\begin{array}{c}- \\
- \\
- \\
- \\
- \\
- \\
5.0-20.0(15.34 \pm 6.33)\end{array}$ & $\begin{array}{c}9(22.5 \%) \\
5(12.5 \%) \\
3(7.5 \%) \\
1(2.5 \%) \\
11(27.5 \%) \\
5.00-9.00(3.325 \pm 1.05) \\
- \\
- \\
3.0-15.0(12.23 \pm 4.55)\end{array}$ & $\begin{array}{l}- \\
- \\
- \\
- \\
- \\
- \\
- \\
-\end{array}$ & $\begin{array}{c}- \\
- \\
- \\
- \\
- \\
- \\
- \\
- \\
0.255\end{array}$ & $\begin{array}{c}- \\
- \\
- \\
- \\
- \\
- \\
- \\
- \\
\mathbf{0 . 0 1 2}\end{array}$ \\
\hline $\begin{array}{l}\text { Frequency of headache attacks; \#/month }(\%) \\
\text { Low-frequency }(<4 / \text { month) } \\
\text { High-frequency ( } \geq 4 / \text { month) }\end{array}$ & $\begin{array}{l}5(8.33 \%) \\
51(85 \%)\end{array}$ & $\begin{array}{c}1(1.67 \%) \\
3(5 \%)\end{array}$ & $\begin{array}{c}4(10 \%) \\
2(5 \%)\end{array}$ & $\begin{array}{l}- \\
-\end{array}$ & - & $\begin{array}{l}- \\
-\end{array}$ \\
\hline BMI; $\mathrm{kg} / \mathrm{m}^{2}$ & $29.07-55.55(39.01 \pm 6.05)$ & $29.07-55.55(28.55 \pm 6.56)$ & $\begin{array}{c}26.20-53.50(30.52 \pm \\
4.59)\end{array}$ & 0.253 & 0.865 & 0.365 \\
\hline $\begin{array}{l}\text { Degree of obesity; \# (\%) } \\
\text { normal: } 18.5-24.9 \mathrm{~kg} / \mathrm{m}^{2} \\
\text { Overweight: } 25.0-29.9 \mathrm{~kg} / \mathrm{m}^{2} \\
\text { Obese: } 30.0-34.9 \mathrm{~kg} / \mathrm{m}^{2} \\
\text { Morbidly obese: }>35.0 \mathrm{~kg} / \mathrm{m}^{2}\end{array}$ & $\begin{array}{c}0 \\
2(3.33 \%) \\
21(35 \%) \\
33(55 \%)\end{array}$ & $\begin{array}{c}0 \\
3(5 \%) \\
1(1.67 \%) \\
0\end{array}$ & $\begin{array}{c}14(35 \%) \\
16(40 \%) \\
2(5 \%) \\
0\end{array}$ & $\begin{array}{l}- \\
- \\
-\end{array}$ & $\begin{array}{l}- \\
- \\
-\end{array}$ & $\begin{array}{l}- \\
- \\
-\end{array}$ \\
\hline WC; $\mathrm{cm}$ & $93.0-136.0(126.27 \pm 9.84)$ & 93.0-136.0 (108.36 \pm 9.99) & $\begin{array}{c}100.0-132.0(87.05 \pm \\
5.32)\end{array}$ & 0.050 & 0.053 & 0.058 \\
\hline $\begin{array}{l}\text { SBP; } \mathrm{mmHg} \\
\text { DBP; } \mathrm{mmHg}\end{array}$ & $\begin{array}{c}110.0-180.0(150.92 \pm \\
16.86) \\
70.0-110.0(105.75 \pm 6.56)\end{array}$ & $\begin{array}{c}100.0-150.0(135.060 \pm \\
16.33) \\
70.0-100.0(80.20 \pm 8.10)\end{array}$ & $\begin{array}{c}100.0-130.0(120.0 \pm 0.0) \\
60.0-85.0(80.0 \pm 0.0)\end{array}$ & $\begin{array}{l}0.052 \\
0.054\end{array}$ & $\begin{array}{l}0.432 \\
0.405\end{array}$ & $\begin{array}{l}0.052 \\
0.050\end{array}$ \\
\hline $\begin{array}{l}\text { Duration of T2DM; years } \\
\text { Duration of obesity; years } \\
\text { Duration of HTN; years }\end{array}$ & $\begin{array}{l}2.0-20.0(5.98 \pm 3.60) \\
2.0-20.0(8.75 \pm 4.47) \\
1.0-18.0(5.12 \pm 3.44)\end{array}$ & $\begin{array}{l}2.0-20.0(6.24 \pm 2.50) \\
2.0-20.0(5.04 \pm 2.08) \\
1.0-18.0(4.92 \pm 2.16)\end{array}$ & $\begin{array}{ll}- \\
- \\
-\end{array}$ & $\begin{array}{l}- \\
- \\
-\end{array}$ & $\begin{array}{c}- \\
0.057 \\
-\end{array}$ & $\begin{array}{l}- \\
- \\
-\end{array}$ \\
\hline $\begin{array}{l}\text { Degree of control on anti-diabetics treatment } \\
\text { Controlled; \# (\%) } \\
\text { Uncontrolled; \# (\%) } \\
\text { Degree of control on anti-hypertensive } \\
\text { treatment } \\
\text { Controlled; \# (\%) } \\
\text { Uncontrolled; \# (\%) } \\
\text { Degree of control on lipid lowering treatment } \\
\text { Controlled; \# (\%) } \\
\text { Uncontrolled; \# }(\%)\end{array}$ & $\begin{array}{c}23(38.33 \%) \\
33(55 \%) \\
\\
24(40 \%) \\
32(53.33 \%) \\
\\
23(38.33 \%) \\
33(55 \%)\end{array}$ & $\begin{array}{l}2(3.33 \%) \\
2(3.33 \%) \\
4(6.67 \%) \\
0\end{array}$ & $\begin{array}{l}- \\
- \\
-\end{array}$ & $\begin{array}{l}- \\
- \\
-\end{array}$ & $\begin{array}{l}- \\
- \\
- \\
-\end{array}$ & $\begin{array}{l}- \\
- \\
- \\
-\end{array}$ \\
\hline
\end{tabular}

Data are expressed as range, mean \pm SD; number (\%); MoA, migraine without aura; MA, migraine with aura; TTH, tension type headache; BMI, body mass index; WC, waist circumference; SBP, systolic blood pressure; DBP, diastolic blood pressure; T2DM, type 2 diabetes mellitus; HTN, hypertension Significance: P1: migraine versus controls; P2: TTH versus controls; P3: migraine versus TTH

Table 1: Demographic, anthropometric and clinical characteristics of the studied groups.

with the cross sectional assessment while clinical evaluation and interviewing with patients and control subjects.

\section{Statistical analysis}

Calculations were done with the statistical package SPSS, version 12.0. Data were presented as mean \pm SD (standard deviation). In tables 1 and 2, non-parametric methods were used to compare the differences between groups (as there were only four subjects in the group of TTH. In tables 3 and 4, one-way ANOVA with a post hoc test was used to compare the differences between the three groups. We used stepwise forward logistic regression to model leptin and headache parameters adjusting for covariates. In patients with MS and migraine to determine whether migraine covariates had relationships to leptin, the logistic regression model was as follow: First, we carried out correlations between dependent variables (frequency and duration of headache) and the independent variables (as BMI, WC, HbAIc, SBP, DBP, TC, TG, LDL-c, HDL-c and leptin). Second, independent variables which had no significant correlations were excluded (after adjustment of age and sex) followed by adjustment of other positively correlated independent variables. For all tests, values of $\mathrm{p}<0.05$ were considered statistically significant.

\section{Results}

This study included 60 patients with MS and complained of headache $($ male $=22$; females $=38)$, with mean age of $47.83 \pm 7.31$ years Nearly $93.33 \%(\mathrm{n}=56)$ had migraine, $58.33 \%(\mathrm{n}=35)$ had episodic migraine $(\mathrm{MoA}=44.64 \%, \mathrm{n}=25 ; \mathrm{MA}=16.6 \%, \mathrm{n}=10), 35 \%(\mathrm{n}=21)$ had chronic migraine and $6.67 \%(\mathrm{n}=4)$ had TTH. In control subjects, migraine was reported in $22.5 \%(\mathrm{n}=9)$ while TTH was reported in $27.5 \%(\mathrm{n}=11)$. The majority of patients had frequent migraine attacks $(91.07 \%, \mathrm{n}=51)$. T2DM and HTN were reported in $100 \%$ of patients, of them $>50 \%$ were uncontrolled on anti-diabetics or anti-hypertensive medications, $91.67(\mathrm{n}=55)$ were obese/morbidly obese, $80 \%(\mathrm{n}=48)$ had hypercholesterolemia and/or hypertriglyceridemia $81.67 \%$ (n $=49)$ had IR and 58.33\% ( $\mathrm{n}=35)$ had hyperliptinemia. Leptin levels were 2.3 fold higher in women compared with men. Demographic, anthropometric, clinical and laboratory characteristics of the studied groups were shown in tables 1 and 2. Compared to patients with TTH, 
Citation: Hamed SA, Ezz-El-Deen ME, Abdou MA (2012) Migraine in Patients with Metabolic Syndrome: Is there a Relationship to Leptin? Metabolomics 2:114. doi:10.4172/2153-0769.1000114

Page 4 of 6

\begin{tabular}{|c|c|c|c|c|c|c|}
\hline \multirow[t]{2}{*}{ Laboratory } & \multicolumn{2}{|c|}{ Patients $(n=60)$} & \multirow[t]{2}{*}{ Control subjects } & \multirow[t]{2}{*}{ P1-value } & \multirow[t]{2}{*}{ P2-value } & \multirow[t]{2}{*}{ P3-value } \\
\hline & $\begin{array}{l}\text { Patients with episodic } \\
\text { and chronic migraine }\end{array}$ & TTH & & & & \\
\hline $\begin{array}{l}\text { Patients with hypercholesterolemia / } \\
\text { dyslipidemia; \# (\%) lipid } \\
\text { TC; mg/dl } \\
\text { LDL-C; mg/dl } \\
\text { TG; mg/dl } \\
\text { HDL-C; mg/dl }\end{array}$ & $\begin{array}{c}48(80 \%) \\
\\
130.20-395.00(229.00 \pm 53.15) \\
58.80-286.60(141.89 \pm \\
44.83) \\
48.40-423.00(189.29 \pm 11.08) \\
17.00-89.00(44.52 \pm 14.78)\end{array}$ & $\begin{array}{c}0 \\
130.20-195.00(130.05 \pm 48.58) \\
58.80-200.00(80.56 \pm 15.50) \\
48.40-200.00(150.85 \pm 23.56) \\
35.00-89.00(39.50 \pm 8.60)\end{array}$ & $\mid \begin{array}{c}0 \\
124.00-195.00(128.10 \pm 70.10) \\
64.00-115.00(57.30 \pm 19.40) \\
49.00-128.00(92.10 \pm 8.20) \\
35.00-48.00(56.90 \pm 1.20)\end{array}$ & $\begin{array}{l}- \\
\mathbf{0 . 0 5 2} \\
\mathbf{0 . 0 0 7} \\
\mathbf{0 . 0 0 6} \\
0.537\end{array}$ & $\begin{array}{l}0.675 \\
0.432 \\
\mathbf{0 . 0 0 1} \\
0.113\end{array}$ & $\begin{array}{c}0.368 \\
\mathbf{0 . 0 0 0 1} \\
0.568 \\
0.067\end{array}$ \\
\hline FBG; mmol/l & $5.12-21.19(10.29 \pm 3.78)$ & $5.35-10.55(10.54 \pm 3.50)$ & $3.10-5.40(4.30 \pm 0.71)$ & 0.0001 & 0.0001 & 0.235 \\
\hline Post-prandial blood glucose; mmol/l & $7.00-16.00(12.69 \pm 2.39)$ & $4.50-16.00(12.59 \pm 5.80)$ & $4.50-6.30(5.37 \pm 0.59)$ & 0.0001 & 0.0001 & 0.432 \\
\hline HbA1c & $5.90-10.90(8.11 \pm 1.22)$ & $2.00-20.10(10.50 \pm 0.25)$ & $4.00-5.70(4.86 \pm 0.48)$ & 0.0001 & 0.0001 & 0.675 \\
\hline Patients with Insulin resistance; \# (\%) & $49(81.67 \%)$ & 0 & 0 & - & - & - \\
\hline Fasting insulin; $\mu \mathrm{lU} / \mathrm{ml}$ & $2.00-35.10(14.17 \pm 10.83)$ & $2.00-10.55(5.56 \pm 1.93)$ & $2.00-7.20(5.56 \pm 1.93)$ & 0.0001 & 0.455 & 0.568 \\
\hline Patients with hyperleptinemia; \# (\%) & $35(58.33 \%)$ & 0 & 0 & - & - & - \\
\hline Leptin; ng/ml & $1.00-106.50(25.32 \pm 7.53)$ & $1.00-60.310(10.60 \pm 4.56)$ & $1.00-22.10(8.49 \pm 6.62)$ & 0.0001 & 0.098 & 0.0001 \\
\hline
\end{tabular}

Data are expressed as mean $\pm \mathrm{SD}$; number $(\%)$

TTH, tension type headache; FBG, fasting blood glucose; HbA1c, glycolysated hemoglobin; TC, total cholesterol; TG, triglycerides; LDL-c, low density lipoprotein cholesterol; HDL-c, high density lipoprotein cholesterol

Significance: P1: migraine versus controls; P2: TTH versus controls; P1: migraine versus TTH

Table 2: Laboratory findings of the studied groups.

\begin{tabular}{|c|c|c|c|c|c|c|c|c|}
\hline Demographic and Clinical characteristics & $\begin{array}{c}\text { MoA } \\
(n=25)\end{array}$ & $\begin{array}{c}\text { MA } \\
(n=10)\end{array}$ & $\begin{array}{c}\text { Chronic migraine } \\
(n=21)\end{array}$ & $\begin{array}{l}\text { TTH } \\
(n=4)\end{array}$ & P1-value & P2-value & P3-value & P4-value \\
\hline Male/female; \# & $9 / 16$ & $2 / 8$ & $8 / 13$ & $1 / 3$ & - & - & - & - \\
\hline Age; years & $47.48 \pm 8.43$ & $49.87 \pm 5.49$ & $49.48 \pm 6.10$ & $48.55 \pm 7.60$ & 0.144 & 0.907 & 0.804 & 0.115 \\
\hline BMI; kg/m² & $39.01 \pm 6.05$ & $36.64 \pm 4.75$ & $39.60 \pm 5.23$ & $28.55 \pm 6.56$ & 0.050 & 0.0 .54 & 0.012 & 0.133 \\
\hline $\begin{array}{l}\text { Degree of obesity; \# (\%) } \\
\text { Normal: } 18.5-24.9 \mathrm{~kg} / \mathrm{m}^{2} \\
\text { Overweight: } 25.0-29.9 \mathrm{~kg} / \mathrm{m}^{2} \\
\text { Obese: } 30.0-34.9 \mathrm{~kg} / \mathrm{m}^{2} \\
\text { Morbidly obese: }>35.0 \mathrm{~kg} / \mathrm{m}^{2}\end{array}$ & $\begin{array}{c}0 \\
2(8 \%) \\
15(60 \%) \\
13(52 \%)\end{array}$ & $\begin{array}{c}0 \\
0 \\
3(30 \%) \\
7(70 \%)\end{array}$ & $\begin{array}{c}0 \\
0 \\
3(14.29 \%) \\
18(85.71 \%)\end{array}$ & $\begin{array}{c}1(1.67 \%) \\
3(5 \%) \\
0 \\
0\end{array}$ & $\begin{array}{l}- \\
- \\
-\end{array}$ & $\begin{array}{l}- \\
- \\
-\end{array}$ & $\begin{array}{l}- \\
- \\
-\end{array}$ & $\begin{array}{l}- \\
- \\
-\end{array}$ \\
\hline WC; $\mathbf{c m}$ & $115.96 \pm 8.84$ & $116.20 \pm 11.85$ & $118.55 \pm 5.67$ & $108.36 \pm 9.99$ & 0.125 & 0.0 .50 & 0.067 & 0.856 \\
\hline $\begin{array}{l}\text { SBP; mmHg } \\
\text { DBP; mmHg }\end{array}$ & $\begin{array}{c}165.80 \pm 10.18 \\
100.20 \pm 4.98\end{array}$ & $\begin{array}{c}150.00 \pm 13.54 \\
95.33 \pm 7.15\end{array}$ & $\begin{array}{c}150.00 \pm 13.33 \\
95.20 \pm 5.20\end{array}$ & $\begin{array}{c}135.060 \pm \\
16.33 \\
80.20 \pm 8.10\end{array}$ & $\begin{array}{l}0.045 \\
0.024\end{array}$ & $\begin{array}{l}0.053 \\
0.050\end{array}$ & $\begin{array}{l}0.050 \\
0.050\end{array}$ & $\begin{array}{l}0.462 \\
0.821\end{array}$ \\
\hline $\begin{array}{l}\text { Duration of T2DM; years } \\
\text { Duration of overweight/obesity; years } \\
\text { Duration of HTN; years }\end{array}$ & $\begin{array}{l}5.92 \pm 3.84 \\
6.72 \pm 3.18 \\
4.60 \pm 2.18\end{array}$ & $\begin{array}{c}5.33 \pm 3.59 \\
10.53 \pm 3.18 \\
6.20 \pm 3.86\end{array}$ & $\begin{array}{l}10.67 \pm 2.56 \\
12.66 \pm 3.56 \\
10.78 \pm 2.40\end{array}$ & $\begin{array}{l}6.24 \pm 2.50 \\
5.04 \pm 2.08 \\
4.92 \pm 2.16\end{array}$ & $\begin{array}{l}0.089 \\
\mathbf{0 . 0 5 3} \\
0.564\end{array}$ & $\begin{array}{c}0.754 \\
\mathbf{0 . 0 0 0 1} \\
0.345\end{array}$ & $\begin{array}{c}0.001 \\
0.0001 \\
0.0001\end{array}$ & $\begin{array}{l}0.675 \\
\mathbf{0 . 0 1 2} \\
0.654\end{array}$ \\
\hline $\begin{array}{l}\text { Degree of control on anti-diabetic treatment } \\
\text { Controlled; \# (\%) } \\
\text { Uncontrolled; \# (\%) } \\
\text { Degree of control on anti-hypertensive treatment } \\
\text { Controlled; \# (\%) } \\
\text { Uncontrolled; \# (\%) } \\
\text { Degree of control on lipid lowering treatment } \\
\text { Controlled; \# (\%) } \\
\text { Uncontrolled; \# (\%) }\end{array}$ & $\begin{array}{l}10(40 \%) \\
15(60 \%)\end{array}$ & $\begin{array}{c}3(30 \%) \\
7(70 \%) \\
0 \\
10(100 \%)\end{array}$ & $\begin{array}{c}12(57.14 \%) \\
9(42.86 \%) \\
\\
5(23.81 \%) \\
16(76.19 \%)\end{array}$ & $\begin{array}{c}3(5 \%) \\
1(1.67 \%)\end{array}$ & - & $\begin{array}{l}- \\
- \\
-\end{array}$ & $\begin{array}{l}- \\
- \\
-\end{array}$ & - \\
\hline
\end{tabular}

Data are expressed as mean $\pm \mathrm{SD}$; number $(\%)$

MoA, migraine without aura; MA, migraine with aura; BMI, body mass index; WC, waist circumference; SBP, systolic blood pressure; DBP, diastolic blood pressure Significance: P1: MoA versus TTH; P2: MA versus TTH; P3: chronic migraine versus TTH; P4: MoA versus MA; P5: MoA versus chronic migraine; P6: MA versus chronicmigraine

Table 3: Demographic, anthropometric and clinical characteristics of the studied groups according to the type of headache

patients with migraine had higher measurements for BMI $(39.01 \pm$ $6.05)$, WC $(\mathrm{P}=0.058)$, poor glycemic control $(8.11 \pm 1.22)$, SBP $(\mathrm{P}=$ $0.052)$, DBP $(P=0.050)$ and serum levels of $L D L-c(P=0.0001)$, fasting insulin $(\mathrm{P}=0.0001)$ and leptin $(\mathrm{P}=0.0001)$. Leptin concentrations were found to be positively correlated with BMI $(\mathrm{r}=0.547, \mathrm{P}=0.008)$, WC $(\mathrm{r}=0.445, \mathrm{P}=0.002), \mathrm{HbAl} \mathrm{c}(\mathrm{r}=0.656, \mathrm{P}=0.001)$ and fasting insulin $(\mathrm{r}=0.613, \mathrm{P}=0.008)$ (tables 3 and 4$)$. After adjusting age and sex, the logistic regression to model leptin and migraine covariates (frequency and duration), leptin concentrations found to correlate with BMI, WC and fasting insulin) but this relationship disappeared after adjustment of these covariates.

\section{Discussions}

The associations between clinical features of migraine and MS and its components have already been reported [1-6]. However, in this study, we reported increased frequency of comorbid migraine in patients with MS and complained of headache (93.33\%), of them, $58.33 \%$ had episodic migraine $(\mathrm{MoA}=44.64 \% ; \mathrm{MA}=16.6 \%), 35 \%$ had chronic 


\begin{tabular}{|c|c|c|c|c|c|c|c|c|}
\hline Laboratory & $\begin{array}{c}\text { MoA } \\
(n=25)\end{array}$ & $\begin{array}{c}\text { MA } \\
(n=10)\end{array}$ & $\begin{array}{c}\text { Chronic migraine } \\
(\mathrm{n}=21)\end{array}$ & $\begin{array}{l}\text { TTH } \\
(n=4)\end{array}$ & P1-value & P2-value & P3-value & P4-value \\
\hline $\begin{array}{l}\text { Lipid } \\
\text { TC; mg/dl } \\
\text { LDL-c; mg/dl } \\
\text { TG; mg/dl } \\
\text { HDL-c; mg/dl }\end{array}$ & $\begin{array}{c}228.54 \pm 42.16 \\
143.39 \pm 40.97 \\
180.38 \pm 10.87 \\
43.54 \pm 14.55\end{array}$ & $\begin{array}{c}210.64 \pm 60.04 \\
122.21 \pm 44.91 \\
207.00 \pm 15.66 \\
46.15 \pm 12.89\end{array}$ & $\begin{array}{c}230.55 \pm 58.98 \\
149.27 \pm 40.70 \\
174.93 \pm 33.69 \\
44.00 \pm 12.99\end{array}$ & $\begin{array}{c}130.05 \pm 48.58 \\
80.56 \pm 15.50 \\
150.85 \pm 23.56 \\
39.50 \pm 8.60\end{array}$ & $\begin{array}{l}\mathbf{0 . 0 0 1} \\
\mathbf{0 . 0 0 1} \\
\mathbf{0 . 0 4 7} \\
0.706\end{array}$ & $\begin{array}{l}0.001 \\
0.001 \\
0.001 \\
0.700\end{array}$ & $\begin{array}{l}\mathbf{0 . 0 0 1} \\
\mathbf{0 . 0 0 1} \\
\mathbf{0 . 0 5 0} \\
0.229\end{array}$ & $\begin{array}{l}0.851 \\
0.056 \\
0.484 \\
0.573\end{array}$ \\
\hline Fasting blood glucose; $\mathrm{mmol} / \mathrm{l}$ & $19.89 \pm 3.74$ & $8.16 \pm 4.52$ & $15.52 \pm 4.22$ & $4.30 \pm 0.71$ & 0.0001 & 0.089 & 0.0001 & 0.001 \\
\hline Post-prandial glucose; $\mathrm{mmol} / \mathrm{l}$ & $21.55 \pm 5.05$ & $13.23 \pm 2.65$ & $20.67 \pm 4.75$ & $5.37 \pm 0.59$ & 0.0001 & 0.546 & 0.0001 & 0.001 \\
\hline HbA1c & $12.35 \pm 1.06$ & $9.5 \pm 0.56$ & $10.50 \pm 0.25$ & $4.86 \pm 0.48$ & 0.0001 & 0.432 & 0.0001 & 0.035 \\
\hline Fasting insulin; $\mu \mathrm{lU} / \mathrm{ml}$ & $15.09 \pm 7.63$ & $12.63 \pm 5.23$ & $13.54 \pm 4.53$ & $5.56 \pm 1.93$ & 0.0001 & 0.0001 & 0.0001 & 0.632 \\
\hline Leptin; $\mathrm{ng} / \mathrm{ml}$ & $27.54 \pm 6.43$ & $30.45 \pm 3.23$ & $30.23 \pm 5.67$ & $8.49 \pm 6.62$ & 0.0001 & 0.0001 & 0.0001 & 0.654 \\
\hline
\end{tabular}

Table 4: Laboratory findings of the studied groups according to the type of headache

migraine of high frequencies (91.07\%), while migraine was reported in $22.5 \%$ of healthy control subjects. Although a $58.33 \%$ prevalence of episodic migraine in patients with MS seems to be different from the numbers reported previously in many studies $(11.9 \%$ in men and $22.5 \%$ in women), this could be explained by use of different study and methodological designs as follow: a) we chose a cut off age of 55 years old, b) all patients had chronic illness, c) the majority of patients were obese/morbidly obese (35\% and 55\% respectively) and, d) the majority of patients with episodic and chronic migraine had higher frequencies of IR ( $81.67 \%)$, higher measurements of SBP and DBP with $>50 \%$ were uncontrolled on anti-hypertensive or anti-diabetic medications.

Here, we reported an increased risk of TBO and abdominal adiposity in patients with episodic and chronic migraine compared to TTH. Large population-based studies indicated that obesity (TBO and/ or abdominal adiposity) is a risk or an exacerbating factor for episodic migraine and migraine progression from episodic to chronic migraine after adjusting for comorbidities, but not for other types of headaches. Obesity is considered as a pro-inflammatory state in which increased inflammatory mediators; vascular hyper reactivity, plasma calcitonin gene-related peptide (CGRP) concentrations and decreased adiponectin concentrations. These alterations may increase the frequency, severity and duration of migraine attacks per se, which in turn would cause central sensitization. Repeated central sensitization may be associated with permanent neuronal damage close to the periaqueductal gray area, with poor modulation to pain and thereafter, chronic migraine [10-13, 20]. In accordance, Horev et al. [10] reported migraine in $48.15 \%$ of the studied morbidly obese women $(\mathrm{MA}=37.04 \%, \mathrm{MoA}=11.11 \%)$ and $14.81 \%$ had TTH. Peres et al. [10] reported headache in $75 \%$ of the studied obese women, of them $66 \%$ had migraine, $9 \%$ had TTH and $48 \%$ had incapacitating headaches. Bigal et al. [20] reported migraine in $22.11 \%$ in and TTH in $1.71 \%$ of studied USA population, of them very frequent headaches (10-14 day/month) was reported in $7.4 \%$ with overweight, $8.2 \%$ in obese and $10.4 \%$ in morbidly obese subjects, compared to $6.5 \%$ with normal weight. Keith et al. [12] in their crosssectional analysis of 11 datasets which included 220,370 adult women ( $\geq 18$ years), the authors identified that a BMI of $\sim 20$ was associated with the lowest risk of headache, BMI of 30 was associated with $\sim 35 \%$ increase in the odds for headache whereas BMI of 40 was associated with $\sim 80 \%$ increase in odds. In this study, we chose a cut off age of 55 years old. In accordance, Peterlin et al. [13] reported increase in the prevalence of migraine with increase in BMI independent to WC in adult's $\leq 55$ years old and regardless of gender and migraine prevalence is increased with increase in WC and independent of BMI in women. While in adults $>55$ years old and regardless of gender, migraine prevalence is not associated with WC or BMI. In addition, migraine prevalence is decreased with WC independent of BMI in women. In fact, in normal individuals, adipose tissue distribution patterns are different in women and men, with younger women having more adipose tissue depots in a gluteofemoral distribution than abdominally, while men of all ages and older women have more abdominal adipose tissue depots than young women [31].

In this study, we reported more poor in glycemic control and higher frequencies of IR in patients with episodic and chronic migraine compared to patients with TTH. In accordance, Rainero et al. [24] found that poor glycemic control (indicated by higher glucose plasma concentrations during OGTT) was associated with evaluated insulin sensitivity in adults with migraine. Several studies have revealed that serum leptin concentrations are not only correlated with BMI and body fat but also with fasting insulin concentrations that is independent of body adiposity [4].

In this study, we reported higher measurements of SBP and DBP in patients with episodic and chronic migraine compared to patients with TTH. In accordance, Scher et al. [32] in their Genetic Epidemiology of Migraine Study which included 5,755 subjects from the Netherlands, the authors found higher blood pressure with migraine compared to TTH. Gudmundsson et al. [26] in their population-based study on 10,366 men and 11,171 women, the authors found that patients with migraine had higher DBP and lower SBP and pulse pressure compared to their matched controls. The authors also found that one standard deviation increase in DBP significantly increased the probability of migraine by $30 \%$ of women compared to $14 \%$ of men, while one standard deviation increase in SBP and pulse pressure significantly decreased the probability of migraine by $19 \%$ and $13 \%$ of men and $25 \%$ and $14 \%$ of women, respectively. It has been identified that poor control of blood pressure may exacerbate the frequency and severity of migraine and patients with chronic migraine are more likely to be hypertensive than patients with episodic headaches. More recently, a unifying view among most recent studies suggests that migraine is positively correlated with DBP but negatively correlated with SBP and pulse pressure. It has been suggested that renin-angiotensin system acts as a biological link between HTN and central nervous system (CNS) activities that are relevant for migraine pathogenesis [27].

In this study, we reported elevated concentrations of TC, TG, LDL-C and low concentrations of HDL-C in patients with episodic and chronic migraine compared to patients with TTH. It has been observed that high levels of free fatty acids, hypercholesterolemia and dyslipidemia are important factors involved in triggering migraine headache [33]. The relationship between abnormal lipid profile and migraine is further supported by the following: a) low fat diet was associated with significant decrease in headache frequency, intensity, duration and medication intake [34], b) high fat diet has been considered to underlie platelet aggregability, which is associated with decreased serotonin and heightened prostaglandin levels leading to vasodilation, the immediate 
precursor of migraine headache [34], and c) increasing dietary intake of polyunsaturated fatty acids have been found to result in formation of less potent inflammatory mediators, attenuate perivascular and neurogenic inflammatory processes and suppress nitric oxide (NO) production, which play roles in migraine pathogenesis [33].

In this study, patients with migraine had higher leptin concentrations compared to patients with TTH. In logistic regression model, leptin did not account for changes in migraine frequency or duration after adjustment for BMI, WC and fasting insulin. This indicates that hyperleptinemia observed in patients with comorbid migraine in patients with MS, is associated with obesity (TBO and abdominal adiposity) and insulin abnormalities. The observed hyperleptinemia among patients implied a state of leptin resistance [35]. It has been suggested that expansion of adipose tissue during weight gain leads to the recruitment of macrophages as well as the synthesis of various mediators by adipocytes as cytokines (TNF- $\alpha$ and IL-6) and adipocytokines (as adiponectin and leptin) which trigger of migraine headache $[36,37]$. The conjoint correlation between leptin and insulin is also supported by finding that leptin has a direct effect on insulin activity and regulation of total body sensitivity to insulin and triglyceride levels in lipodystrophic syndromes [37]. Hyperleptinemia in diabetic and obese subjects might be due to the stimulatory effect of insulin on adipose tissues stimulating more expression of obesity gene and more leptin secretion [37,38]. Therefore, the relationship between leptin and insulin should be revealed through their metabolic effects on endocrine disorders and MS. In contrast, Haffner et al. [38] reported low inter-ictal leptin concentrations in patients with migraine compared to their control subjects when unadjusted for fat mass. The authors suggested that hyperleptinemia and hyperinsulinemia might be due to the effect of oral hypoglycemic drugs (as sulphonyl urea and glibenclamide). These drugs are reported to increase the circadian leptin and insulin concentrations.

However, and despite the strength of our findings, this study had a main limitation, which is the higher frequency of migraine (3-fold increase in the prevalence of migraine compared to many previous studies). It might be due to small sample size which may create sample distortion bias. However, we explain this as follow: 1) our main aim was to determine the relationship between MS, leptin and migraine in a group of patients with MS and complain with headache, 2) we chose a cut off age of 55 years old, 3) our majority of patients were MS were obese/morbidly obese, had higher frequencies of IR, had higher measurements of SBP and DBP, with $>50 \%$ were uncontrolled on anti-diabetic and anti-hypertensive medications and all had chronic illnesses (migraine) and MS and its components

\section{Conclusion}

We conclude that: migraine is a very frequent comorbidity associated with MS. It mainly related to obesity (TBO and abdominal adiposity T2DM, IR HTN and hypercholesterolemia/dyslipidemia) Hyperleptinemia observed in patients with MS and having migraine is associated with obesity (TBO and abdominal adiposity) and insulin abnormalities. Delineation of migraine comorbidity in MS is important, because it can help to improve treatment strategies and the understanding of the possible pathophysiology of migraine.

\section{Conflicts of Interest}

We declare that this work has no conflict of interests. There is no involvement of sponsor for this work design, data collection, analysis, interpretation, drafting, nor the decision to submit this paper for publication. All are authors' responsibility.

\section{References}

1. Ford ES, Giles WH, Dietz WH (2002) Prevalence of the metabolic syndrome among US adults: findings from the third National Health and Nutrition Examination Survey. JAMA 287: 356-359.

2. Steinbaum SR (2004) The metabolic syndrome: an emerging health epidemic in women. Prog Cardiovasc Dis 46: 321-336.

3. Alberti KG, Eckel RH, Grundy SM, Zimmet PZ, Cleeman Jl, et al. (2009) Harmonizing the metabolic syndrome: a joint interim statement of the International Diabetes Federation Task Force on Epidemiology and Prevention National Heart, Lung, and Blood Institute; American Heart Association; World Heart Federation; International Atherosclerosis Society; and International Association for the Study of Obesity. Circulation 120: 1640-1645.

4. Wang J, Liu R, Hawkins M, Barzilai N, Rossetti L (1998) A nutrient-sensing pathway regulates leptin gene expression in muscle and fat. Nature 393: 684688

5. Guldiken B, Guldiken S, Taskiran B, Koc G, Turgut N (2009) Migraine in metabolic syndrome. Neurologist 15: 55-58

6. Takeshima T (2009) Metabolic syndrome and prevention of migraine headache Brain Nerve. 61: 1143-1153

7. Lipton RB, Stewart WF, Diamond S, Diamond ML, Reed M (2001) Prevalence and burden of migraine in the United States: data from the American Migraine Study II. Headache 41: 646-657.

8. Headache Classification Subcommittee of the International Headache Society (2004) The International Classification of Headache Disorders: 2nd edition. Cephalalgia 1: 9-160

9. Goadsby PJ, Lipton RB, Ferrari MD (2002) Migraine-current understanding and treatment. N Engl J Med 346: 257-270.

10. Horev A, Wirguin I, Lantsberg L, Ifergane G (2005) A high incidence of migraine with aura among morbidly obese women. Headache 45: 936-938.

11. Peres MF, Lerário DD, Garrido AB, Zukerman E (2005) Primary headaches in obese patients. Arq Neuropsiquiatr 63: 931-933.

12. Keith SW, Wang C, Fontaine KR, Cowan CD, Allison DB (2008) BMI and headache among women: Results from 11 epidemiologic datasets. Obesity 16 : 377-383.

13. Peterlin BL, Rosso AL, Rapoport AM, Scher Al (2010) Obesity and migraine: the effect of age, gender and adipose tissue distribution. Headache 50: 52-62.

14. Groop LC, Bonadonna RC, DelPrato S, Ratheiser K, Zyck K, et al. (1989) Glucose and free fatty acid metabolism in non-insulin-dependent diabetes mellitus: evidence for multiple sites of insulin resistance. J Clin Invest 84: 205 213

15. Ford ES, Abbasi F, Reaven GM (2005) Prevalence of insulin resistance and the metabolic syndrome with alternative definitions of impaired fasting glucose. Atherosclerosis 181: 143-148.

16. Cirillo M, Stellato D, Lombardi C, De Santo NG, Covelli V (1999) Headache and cardiovascular risk factors: positive association with hypertension. Headache 39: $409-416$

17. Garaulet M, Pérez-Llamas F, Zamora S, Tebar FJ (2002) Interrelationship between serum lipid profile, serum hormones and other components of the metabolic syndrome. J Physiol Biochem 58: 151-160.

18. Harwood HJ Jr (2012) The adipocyte as an endocrine organ in the regulation of metabolic homeostasis. Neuropharmacology 63: 57-75

19. Elmquist JK, Elias CF, Saper CB (1999) From lesions to leptin: hypothalamic control of food intake and body weight. Neuron 22: 221-232.

20. Bigal ME, Lipton RB, Holland PR, Goadsby PJ (2007) Obesity, migraine, and chronic migraine: possible mechanisms of interaction. Neurology 68: 1851 1861.

21. Esteghamati A, Noshad S, Khalilzadeh O, Morteza A, Nazeri A, et al. (2011) Contribution of serum leptin to metabolic syndrome in obese and non obese subjects. Arch Med Res 42: 244-251.

22. Stelzer I, Zelzer S, Raggam RB, Prüller F, Truschnig-Wilders M, et al. (2012 Link between leptin and interleukin- 6 levels in the initial phase of obesity related inflammation. Transl Res 159:118-124. 
Citation: Hamed SA, Ezz-El-Deen ME, Abdou MA (2012) Migraine in Patients with Metabolic Syndrome: Is there a Relationship to Leptin? Metabolomics 2:114. doi:10.4172/2153-0769.1000114

23. Al-Shoumer KA, Al-Asousi AA, Doi SA, Vasanthy BA (2008) Serum leptin and its relationship with metabolic variables in Arabs with type 2 diabetes mellitus. Ann Saudi Med 28: 367-370.

24. Rainero I, Limone P, Ferrero M, Valfrè W, Pelissetto C, Rubino E, Gentile S, Lo Giudice R, Pinessi L (2005) Insulin sensitivity is impaired in patients with migraine. Cephalgia 25: 593-597.

25. Esteghamati A, Khalilzadeh O, Anvari M, Rashidi A, Mokhtari M (2009) Association of serum leptin levels with homeostasis model assessmentestimated insulin resistance and metabolic syndrome: the key role of central obesity. Metab Syndr Relat Disord 7: 447-452.

26. Gudmundsson LS, Thorgeirsson G, Sigfusson N, Sigvaldason H, Johannsson M (2006) Migraine patients have lower systolic but higher diastolic blood pressure compared with controls in a population-based study of 21,537 subjects. The Reykjavik Study. Cephalgia 26: 436-444.

27. Paterna S, Di Pasquale P, D'Angelo A, Seidita G, Tuttolomondo A, et al. (2000) Angiotensin-converting enzyme gene deletion polymorphism determines an increase in frequency of migraine attacks in patients suffering from migraine without aura. Eur Neurol 43: 133-136.

28. Monastero R, Pipia C, Cefalù AB, Liveri ET, Rosano R, et al. (2008) Association between plasma lipid levels and migraine in subjects aged $>$ or $=50$ years: preliminary data from the Zabùt Aging Project. Neurol Sci 29: S179-181.

29. Hamed SA, Hamed EA, Ezz Eldin AM, Mahmoud NM (2010) Vascular risk factors, endothelial function and carotid thickness in patients with migraine: relationship to atherosclerosis. J stroke cerebrovascular dis 19: 92-103.

30. Niewoehner CB (1998) Endocrine pathophysiology. Fence Ceek Publ, Madison
31. Matthews DR, Hosker JP, Rudenski AS, Naylor BA, Treacher DF, et al. (1985) Homeostasis model assessment: insulin resistance and $\beta$-cell function from fasting plasma glucose and insulin concentrations in men. Diabetologia 28 : 412-419.

32. Scher Al, Terwindt GM, Picavet HS, Verschuren WM, Ferrari MD, et al. (2005) Cardiovascular risk factors in migraine: The GEM population-based study. Neurology 64: 614-620.

33. Harel Z, Gascon G, Riggs S, Vaz R, Brown W, et al. (2002) Supplementation with Omega-3 polyunsaturated fatty acids in the management of recurrent migraines in adolescents. J Adolesc Health 31: 154-161.

34. Bic Z, Blix GG, Hopp HP, Leslie FM, Schell MJ (1999) The influence of a low fat-diet on incidence and severity of migraine headaches. J Women's Heath Gender-based Med 8: 623-630.

35. Kellerer M, Lammers R, Fritsche A, Strack V, Machicao F, et al. (2001) Insulin inhibits leptin receptor signalling in HEK293 cells at the level of janus kinase-2 a potential mechanism for hyperinsulinaemia-associated leptin resistance. Diabetologia 44: 1125-1132.

36. Hotta K, Funahashi T, Arita Y, Takahashi M, Matsuda M, et al. (2000) Plasma concentrations of a novel adipose-specific protein, adiponectin, in type 2 diabetes patients. Arterioscler Thromb Vasc Biol 20: 1595-1599.

37. Nagasaka S, Ishikawa S, Nakamura T, Kawakami A, Rokkaku K, et al. (1998) Association of endogenous insulin secretion and mode of therapy with body fat and serum leptin levels in diabetic subjects. Metabolism 47: 1391-1396.

38. Haffner SM, Gingerich RL, Miettinen H, Stern MP (1996) Leptin concentrations in relation to overall adiposity and regional body fat distribution in Mexican Americans. Int J Obes Relat Metab Disord 20: 904-908. 